\title{
FSTL1 aggravates sepsis-induced acute kidney injury through regulating TLR4/MyD88/NF- $\kappa$ B pathway in newborn rats
}

\author{
Chuanlong Song ${ }^{1}$, Ayinuerguli Adili ${ }^{1}$, Adilijiang Kari ${ }^{1}$, Abulaiti Abuduhaer ${ }^{1, \star}$
}

\author{
${ }^{1}$ Department of Paediatric Intensive \\ Care Unit (PICU), the First Affiliated \\ Hospital of Xinjiang Medical University, \\ 830054 Urumqi City, Xinjiang Uygur \\ Autonomous Region, China \\ *Correspondence \\ Abulaiti63@163.com \\ (Abulaiti Abuduhaer)
}

\begin{abstract}
Background: The aim of this work was to investigate whether Follistatin like 1 (FSTL1) exerted an effect on acute kidney injury (AKI) induced by sepsis, and to explore the molecular mechanism.

Methods: A cecal ligation and puncture (CLP) model was established and adenoviral solution was administrated to newborn rats to achieve FSTL1 knockdown. Colorimetric assays measured concentrations of serum creatinine and blood urea nitrogen (BUN) and ELISA assays were performed to examine TNF- $\alpha$, IL- $1 \beta$ and IL- 6 levels in serum and kidney tissues. Kidney histological analysis was performed using hematoxylin-eosin (HE) staining. Protein levels of toll-like receptor 4 (TLR4), myeloid differentiation primary response 88 (MyD88), phosphorylated p65 (p-p65) and total p65 (p65) were determined by western blotting.

Results: FSTL1 was significantly up-regulated in kidneys following CLP, but subsequent FSTL1 inhibition alleviated AKI. FSTL1 knockdown following CLP inhibited the production of TNF- $\alpha$, IL- $1 \beta$, IL- 6 and inactivated the TLR4/MyD88/NF$\kappa \mathrm{B}$ pathway. Furthermore, FSTL1 overexpression activated the TLR4/MyD88/NF- $\kappa \mathrm{B}$ pathway following CLP, but TLR4 inhibitor TAK242 abolished this effect.

Conclusions: FSTL1 aggravates sepsis-induced acute kidney injury through regulating the TLR4/MyD88/NF- $\kappa$ B pathway.
\end{abstract}

\section{Keywords}

FSTL1; Sepsis; Acute kidney injury; NF- $\kappa \mathrm{B}$

\section{Introduction}

Sepsis has been identified as a common clinical disease in pediatric intensive care units (PICU) with a high mortality rate [1]. Sepsis is caused by an inflammatory response instigated by the host's imbalance of bioactive chemicals produced by infectious pathogens [1]. Once sepsis occurs, a large number of toxins, inflammatory mediators, and metabolites are produced, leading to sepsis with acute respiratory distress syndrome, cardiovascular dysfunction, or even multiple organ dysfunction [2]. Acute kidney injury (AKI) is one of the syndromes caused by sepsis [3]. The pathophysiological mechanism of AKI is complicated, with renal microcirculation disorder, inflammatory injury and an adaptive response by the renal tubular epithelial cells participating in the overall process [3]. Even though the general responses have been characterized, the molecular mechanism has not yet been determined.

Toll-like receptors (TLRs) dominate the innate immune receptors which respond to pathogen-related molecular patterns (PAMPs) [4]. TLRs not only initiate primary responses to invading pathogens, but also induce adaptive immune responses [5]. Toll-like receptor 4 (TLR4) is a member of the pat- tern recognition receptor family, which induce inflammatory responses by recognizing endogenous ligands related to tissue damage, this is especially important for antigen-specific adaptive immune responses [6]. Myeloid differentiation primary response 88 (MyD88) has been identified as a universal adapter protein, which usually induces production of multiple inflammatory cytokines and activation of NF- $\kappa \mathrm{B}$ [7]. Castoldi et al. [8] proved that inhibition of TLR4/MyD88 signaling attenuates LPS-induced AKI. This indicates that regulation of the TLR4/MyD88/NF- $\kappa$ B pathway may present as a promising strategy to treat AKI.

Follistatin like 1 (FSTL1) is a secreted glycoprotein that contains a follistatin domain and an extracellular calciumbinding domain [9]. A large number of reports have indicated that FSTL1 is involved in regulating inflammation responses [10]. For example, FSTL1 aggravates arthritis in rats by promoting the expression of inflammatory cytokines [11], whilst FSTL1 inhibition suppresses oxLDL-induced inflammation via the TLR4/MyD88/NF- $\kappa \mathrm{B}$ and MAPK axis [12]. However, whether FSTL1 exerts an effect on AKI induced by sepsis has yet to be reported.

In the current study, the effects of FSTL1 on AKI induced by 
A

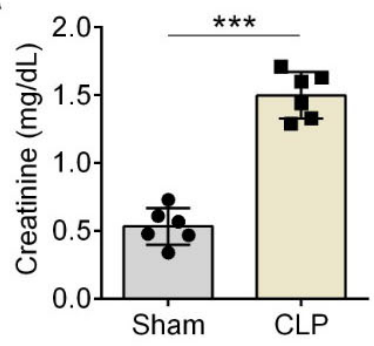

D

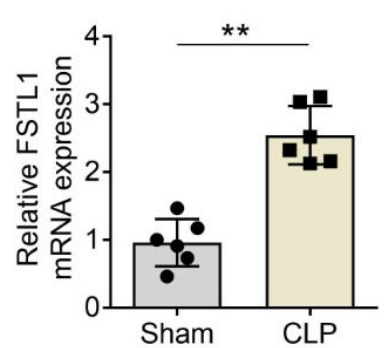

B

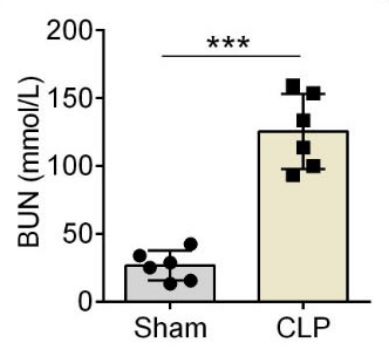

$E$

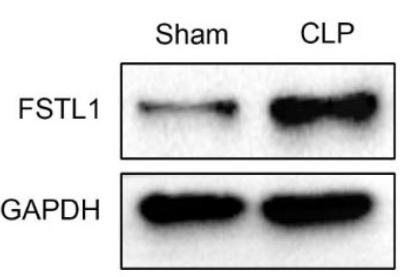

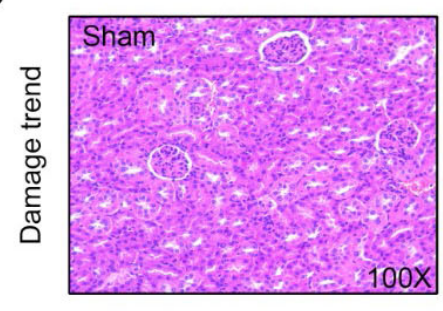

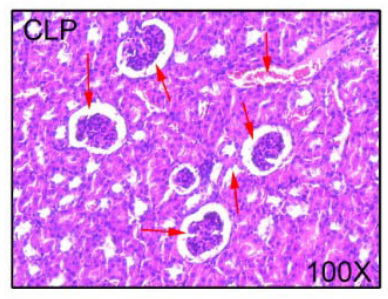

F I G U R E 1. FSTL1 is significantly up-regulated in kidney after CLP. (A) and (B) The levels of creatinine and blood urea nitrogen (BUN) in serum were examined using commercially available kits. (C) Hematoxylin-eosin (HE) staining was performed and histological analysis showed tubular degeneration and vacuolization. Scale bars: $100 \times$. (D) The FSTL1 mRNA levels were detected using qRT-PCR, and (E) protein levels were measured using western blotting. ${ }^{* *} p<0.001,{ }^{* * *} p<0.001$.

sepsis, and the corresponding molecular mechanisms involved were investigated.

\section{Materials and methods}

\subsection{Establishment of sepsis model}

Cecal ligation and puncture (CLP) was performed to establish a sepsis model. Briefly, the newborn rats (3-8 days and 8-14 $\mathrm{g}$ in weight), from distinct littermates, were randomly assigned to each group (6 rats per group). After each rat was anesthetized, a $1 \mathrm{~cm}$ incision was cut along the midline of the anterior abdomen. The epidermis and muscle layer were separated and the cecum was isolated. Thereafter the cecum was ligated with No. 3 thread at $1 / 2$ of the cecum. Following ligation, a No. 8 needle was used to the puncture a hole about $0.5 \mathrm{~cm}$ from the end of the cecum, then the muscle layer and skin were sutured. Rats of sham group were performed with the same operations as CLP but without ligation and puncture of the cecum. Following surgery, the rats received fluid resuscitation with $0.25 \mathrm{~mL}$ normal saline injected subcutaneously and bloods samples were collected 24 hours post-operatively. Kidney samples were divided in half to enable both histological analysis and western blot and ELISA analysis. All of the experimental procedures and animal care were approved by the Ethics Committee of the First Affiliated Hospital of Xinjiang Medical University (Approval No. 20200301-09) and performed according to the Guide for the Care and Use of Laboratory Animals [13].

\subsection{Adenovirus and TAK242 administration}

Adenoviral solution expressing shRNA targeting FSTL1 (5'-GGACGAAACCTATGCAGATGG-3') and adenoviral solution for FSTL1 overexpression were purchased from GeneChem (Shanghai, China). For adenovirus administration, the left kidney of each rat was exposed via a flank incision and the viral solution was administrated by microinjection into three places of the renal vein. TAK242 ( $3 \mathrm{mg} / \mathrm{kg}$ body weight) or its vehicle (DMSO) were administrated via intraperitoneal injection.

\subsection{Western blotting}

Western blots were performed as previously described [14]. The primary antibodies used consisted of TLR4 (\#14358; 1 : 1000), MyD88 (\#4283; 1 : 1000), phosphorylated p65 (pp65; \#3033; 1 : 1000), total p65 (p65; \#8242; 1 : 1000) and GAPDH (\#5174; $1: 1000)$, which were obtained from Cell Signaling Technology (MA, USA).

\subsection{ELISA}

ELISA was performed as per the instructions in the commercially available kits. TNF- $\alpha$ (\# KRC3011), IL-1 $\beta$ (\# BMS630) and IL-6 (\# BMS625) levels in both the serum and kidney tissue were tested using these ELISA kits which obtained from Thermo Fisher Scientific (USA).

\subsection{Measurement of creatinine and blood urea nitrogen (BUN)}

Colorimetric assays were used to detect the concentrations of serum creatinine and blood urea nitrogen (BUN) using commercially available kits. The creatinine (serum) colorimetric assay and urea nitrogen (BUN) colorimetric detection kits were purchased from Cayman Chemical (MI, USA) and Thermo Fisher Scientific (USA) respectively. 

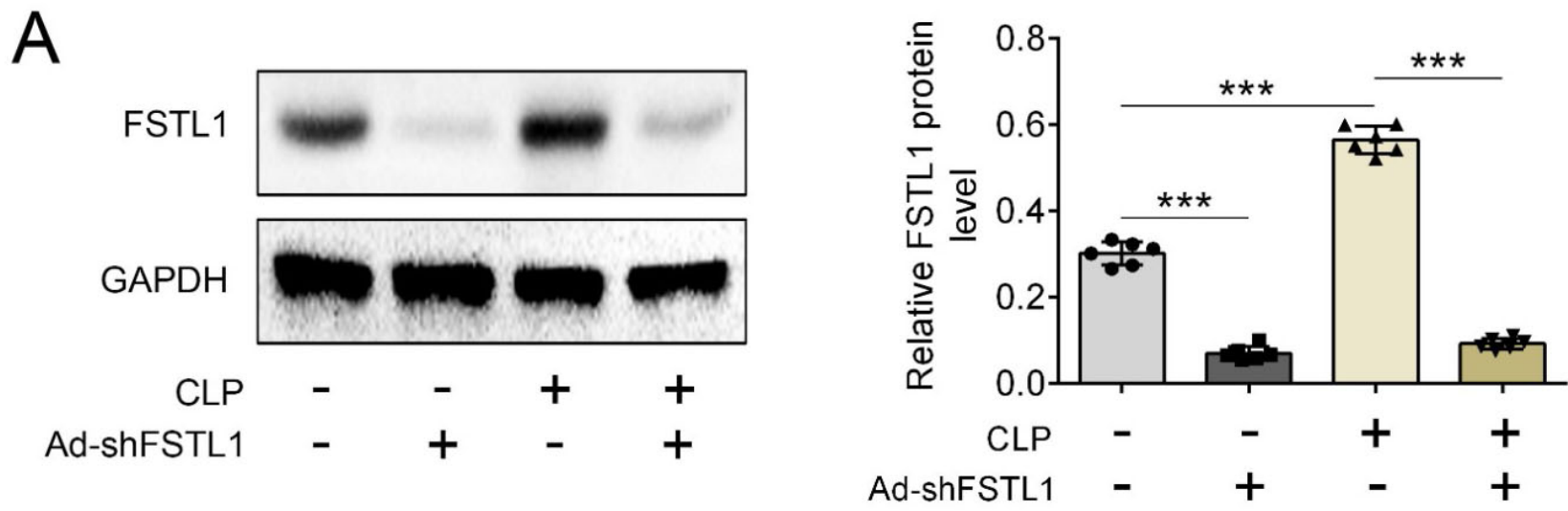

B
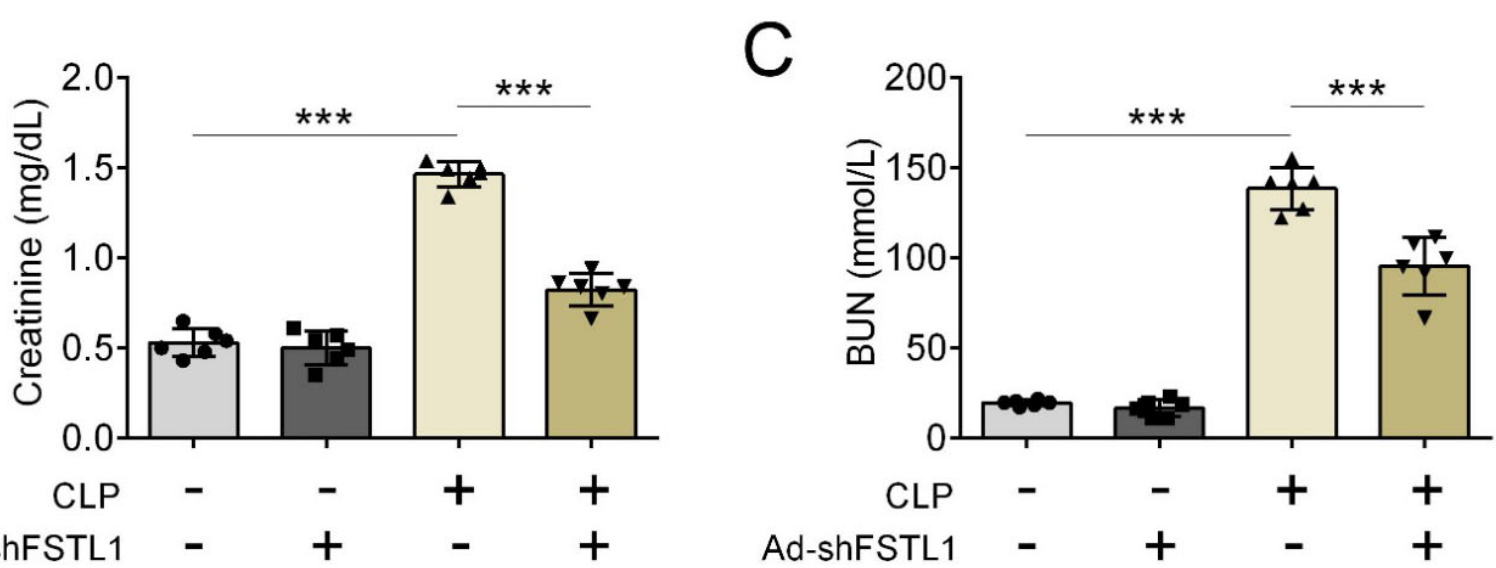

D
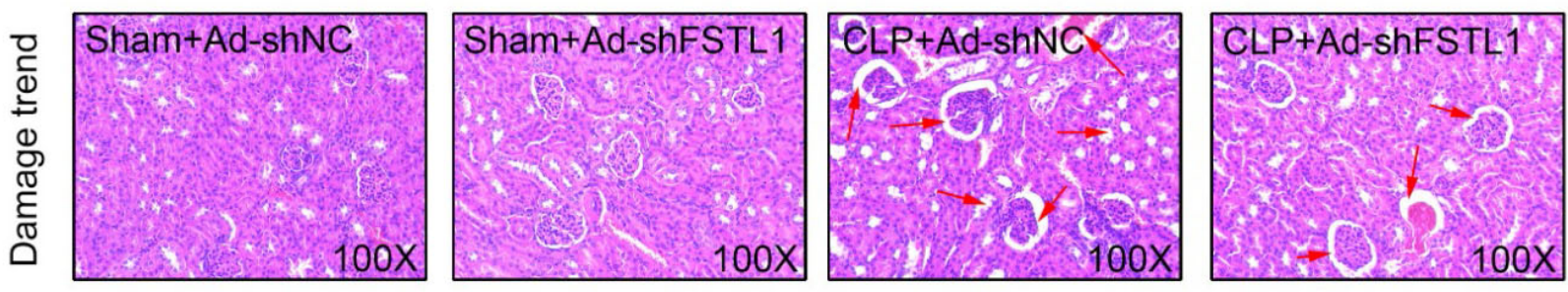

F I G U R E 2. FSTL1 inhibition alleviates acute kidney injury after CLP. (A) An adenoviral vector expressing shRNA targeting FSTL1 was injected into rats prior to CLP to achieve FSTL1 knockdown, FSTL1 protein levels were quantified via western blotting. (B) and (C) show the levels of creatinine and blood urea nitrogen (BUN) in serum. (D) HE staining was performed for histological analysis showing tubular degeneration and vacuolization. Scale bars: $100 \times . * * * p<0.001$.

\subsection{Hematoxylin-eosin (HE) staining}

Kidney tissues from the different treatment groups were harvested and fixed in 4\% formaldehyde. Hematoxylin-eosin (HE) staining was performed for histological analysis of kidney tissues using a previously described protocol [15].

\subsection{Quantitative real-time PCR (qRT-PCR)}

Total RNA extraction was performed using TRIzol@ Reagent (Invitrogen, USA). In total $1 \mu \mathrm{g}$ of RNA was reversetranscribed into cDNA using M-MLV Reverse Transcriptase (Invitrogen, USA). PCR reactions were performed using the CFX96 Real-Time PCR system (Bio-Rad, Hercules, CA,
USA) with the $2 \times$ SYBR Green qPCR Master Mix (Bimake, USA). The primer pair used for FSTL1 amplification was:

\section{5'-AAGAAGAGGCACGTAGCACC-3' (forward) and 5'-TGGTGATTCGGTGATTGCCA-3' (reverse).}

\subsection{Statistical analysis}

All the data were shown as the mean \pm standard deviation (SD). GraphPad Prism 7.0 software was used to analyze the data using an independent student's $t$-test. A value of $p<0.05$ was considered statistically significant. 
A

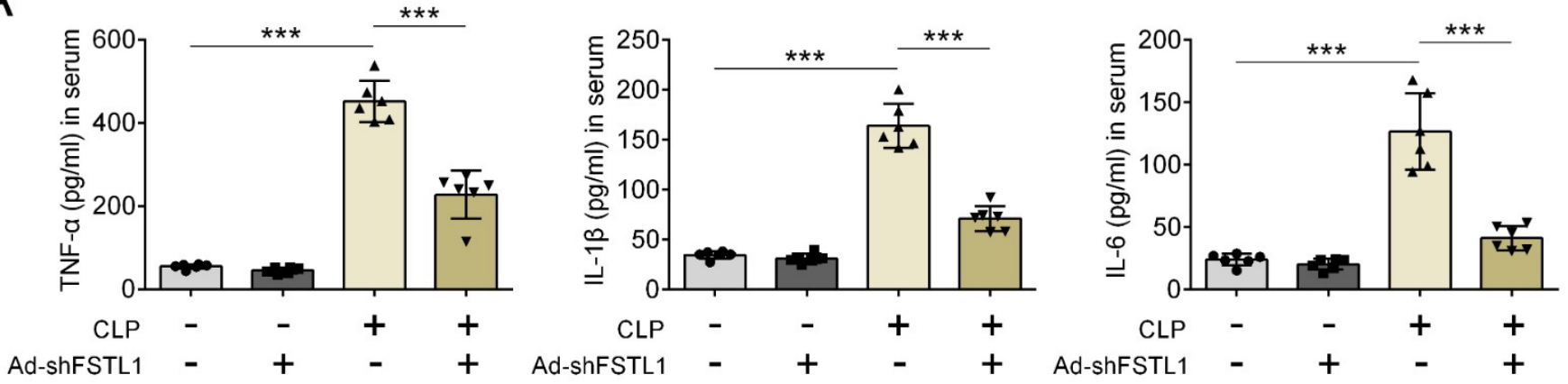

B
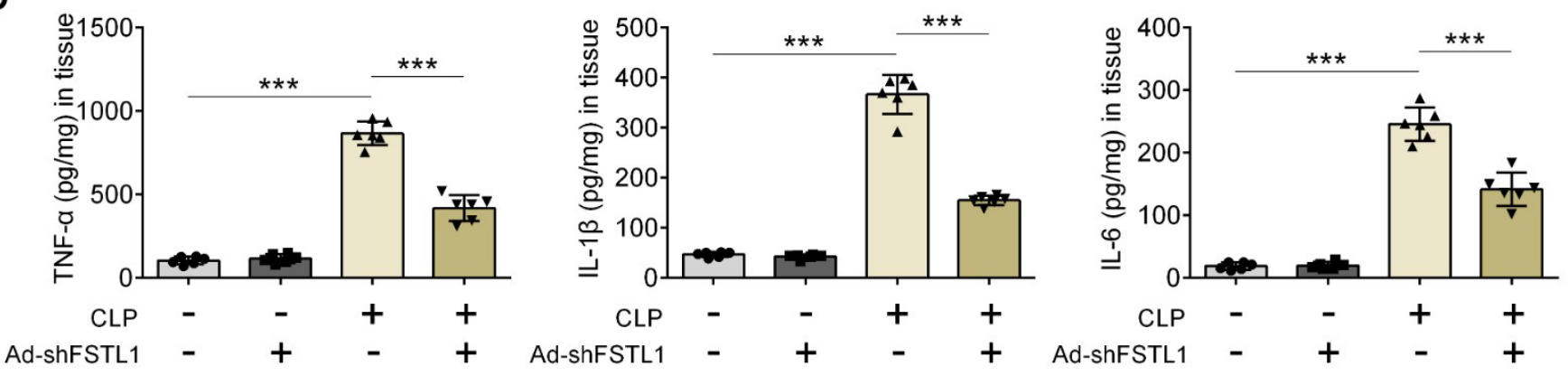

F I G U R E 3. FSTL1 inhibition relieves inflammation after CLP. (A) Proinflammatory cytokines including TNF- $\alpha$, IL-1 $\beta$, and IL- 6 in serum with different treatments were examined by ELISA. (B) The levels of TNF- $\alpha$, IL-1 $\beta$, and IL- 6 in kidney tissues under the differing treatment regimes were also tested by ELISA. $* * * p<0.001$.

\section{Results}

\subsection{FSTL1 was significantly up-regulated in kidney tissue following CLP}

To investigate the role of follistatin like 1 (FSTL1) in sepsisinduced acute kidney injury (AKI), a cecal ligation and puncture (CLP) model was established. Then the levels of creatinine and blood urea nitrogen (BUN) in serum were examined. Creatinine and BUN levels were significantly increased in the CLP group compared to the sham group (Fig. 1A,B). In addition, histological analysis performed on the hematoxylineosin (HE) stained tissue showed aggravated tubular degeneration and vacuolization in the CLP group (Fig. 1C). Moreover, both mRNA and protein levels of FSTL1 were significantly increased in the CLP group (Fig. 1D,E), which indicated that FSTL1 may be involved in the pathogenesis of sepsis-induced AKI.

\subsection{FSTL1 inhibition alleviates acute kidney injury after CLP}

An adenoviral vector expressing shRNA targeting FSTL1 was injected to a subsequent group of rats prior to CLP, in order to achieve FSTL1 knockdown (Fig. 2A). Then AKI samples were evaluated the results demonstrated that levels of serum creatinine and BUN were remarkably increased in the CLP group, but inhibition of FSTL1 significantly relieved kidney injuries following exposure to CLP (Fig. 2B,C). Significant improvement in kidney histology with alleviated tubular degeneration and vacuolization was also observed following FSTL1 knock- down (Fig. 2D). These data suggested that FSTL1 inhibition alleviates acute kidney injury caused by CLP.

\subsection{FSTL1 inhibition relieves inflammation after CLP}

To further determine the mechanism by which FSTL1 regulates the progress following exposure to sepsis-induced AKI, proinflammatory cytokines including TNF- $\alpha$, IL- $1 \beta$, and IL-6 were examined in both serum and kidney tissues from the rats. The levels of TNF- $\alpha$, IL- $1 \beta$, and IL- 6 were all significantly increased in both the serum and kidney tissues following the CLP procedure, however FSTL1 knockdown significantly abolished this effect (Fig. 3A,B). These results indicated that FSTL1 inhibition alleviates acute kidney injury by reducing inflammation resulting from CLP.

\subsection{FSTL1 inhibition alleviates sepsis-induced acute kidney injury by regulating the TLR4/MyD88/NF- $\kappa$ B pathway}

To further investigate the mechanisms involved following AKI, TLR4, MyD88, phosphorylated p65 (p-p65) and total p65 (p65) protein levels were detected using western blotting. The levels of TLR4, MyD88 and p-p65/p65 dramatically decreased when FSTL1 was inhibited, but significantly increased in kidneys exposed to CLP (Fig. 4A). Furthermore, FSTL1 knockdown prior to CLP significantly rescued the dysregulation of the TLR4/MyD88/NF- $\kappa$ B signaling pathway (Fig. 4A). In addition, protein expression of TLR4, MyD88, p-p65 and p65 in CLP rats treated with Ad-FSTL1 for FSTL1 

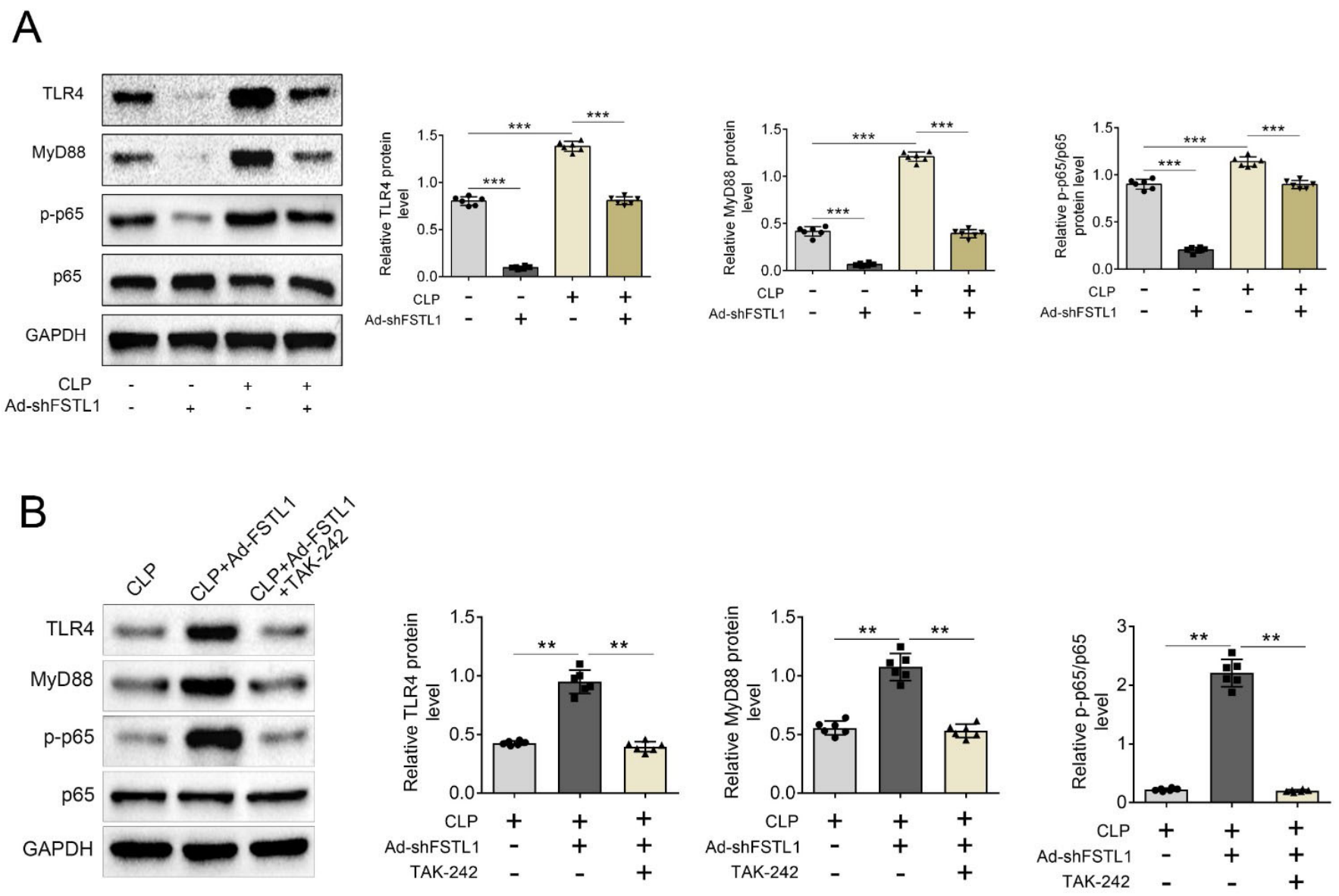

FIGURE 4. FSTL1 inhibition alleviates sepsis-induced acute kidney injury by regulating the TLR4/MyD88/NF$\kappa$ B pathway. (A) Protein expression of toll-like receptor 4 (TLR4), myeloid differentiation primary response 88 (MyD88), phosphorylated p65 (p-p65) and total p65 (p65) in groups with CLP or Ad-shFSTL1 treatment were detected by western blotting. (B) Protein expression of TLR4, MyD88, p-p65 and p65 in CLP rats treated with Ad-FSTL1 or the TLR4 inhibitor TAK242 were detected using western blotting. Results were quantified by ImageJ software. ${ }^{* *} p<0.001,{ }^{* * *} p<0.001$.

overexpression, or the TLR4 inhibitor TAK242, were detected. FSTL1 overexpression activated the TLR4/MyD88/NF- $\kappa$ B pathway following CLP, but TLR4 inhibitor TAK242 abolished this effect. The results further supported the hypothesis that TLR4 signaling mediated the effects of FSTL1 on sepsis-induced AKI (Fig. 4B). Collectively, FSTL1 inhibition alleviates sepsis-induced AKI by regulating the TLR4/MyD88/NF- $\kappa$ B pathway.

\section{Discussion}

Sepsis is a common disease in pediatric intensive care units (PICU) and sepsis-induced acute kidney injury (AKI) is a frequently observed complication with a high mortality rate. Renal microcirculation disorder, inflammatory injury, and adaptive response of renal tubular epithelial cells have been identified as the main causes of sepsis-induced AKI [3]. Inhibition of cytokine production and reduction of inflammation are reported to be promising strategies for treating sepsisinduced AKI [16]. In the current study, the data indicated that FSTL1 is significantly up-regulated in kidneys exposed to CLP. Furthermore, FSTL1 inhibition alleviates acute kidney injury after CLP, and relieves inflammation via regulation of the TLR4/MyD88/NF- $\kappa$ B pathway.
The role of Follistatin like 1 (FSTL1) has been identified in a variety of diseases [17]. In the current study, the effect of FSTL1 on AKI is explored. A previously published study indicated that FSTL1 promotes the production of IL- $1 \beta$ and TNF- $\alpha$ in macrophages within cases of nasopharyngeal carcinoma [18]. Additionally, FSTL1 facilitates enhanced levels of IL- $1 \beta$, TNF- $\alpha$ and IL- 6 in chondrocytes and activates the NF- $\kappa$ B pathway in osteoarthritis [11]. FSTL1 also exacerbates lung injury via induction of IL- $1 \beta$ production by macrophages and is mediated by the NLRP3 inflammasome [19]. Roles for FSTL1 have also been implicated in cardiovascular diseases such as ischemia/reperfusion (I/R) injury and valvular heart disease. However, FSTL1 has also been shown to have a protective effect during cardiovascular disease by reducing cardiomyocyte apoptosis [17]. In the present study, FSTL1 is first proven to be involved in AKI and induces the production of pro-inflammatory cytokines including TNF- $\alpha$, IL- $1 \beta$, and IL-6. Our results are consistent with the findings that FSTL1 serves as a pro-inflammatory factor, which promotes AKI.

Accumulated evidence has suggested that the TLR4/MyD88/NF- $\kappa \mathrm{B}$ axis is an important signal pathway that regulates inflammatory response-mediated injury in a variety of diseases. Renal $I / R$ injury following kidney transplantation is always induced by oxidative stress and 
excessive inflammatory cytokines due to the activation of TLR4/MyD88/NF- $\kappa$ B axis [20]. Dexmedetomidine treatment inactivates TLR $4 / \mathrm{NF}-\kappa \mathrm{B}$ and attenuates the AKI observed after liver transplantation [21]. In addition, dioscin inhibits renal $\mathrm{I} / \mathrm{R}$ injury by down-regulating the TLR4/MyD88 expression mediated by HSP70 [22]. Interestingly, TLR4/MyD88/NF- $\kappa \mathrm{B}$ signaling has also been widely studied for its role in kidney injury, and astilbin, glutamine and LIGHT contribute to kidney injury and inflammatory responses as a result of TLR4/MyD88/NF- $\kappa \mathrm{B}$ signaling pathway down-regulation [23-25]. In the present study, protein levels of TLR4, MyD88, phosphorylated p65 (p-p65) and total p65 (p65) were examined to comprehend the mechanism by which FSTL1 is involved in sepsis-induced AKI. Indeed, FSTL1 exerts its effect on sepsis-induced AKI by regulating TLR4/MyD88/NF- $\kappa \mathrm{B}$ signaling. However, the specific target and molecular mechanism was not completely elucidated or clarified in the current study. We therefore intend to investigate and disclose the downward targets of FSTL1 in future studies.

TLR4 inhibitor TAK-242 has a protective effect on AKI by targeting TLR4/MyD88/NF- $\kappa \mathrm{B}$ signaling pathway. In this study, TAK-242 administration rescued the overactivated TLR4/MyD88/NF- $\kappa$ B pathway owing to FSTL1 overexpression. Therefore, targeting FSTL1 is probably a potential treatment strategy of AKI. In conclusion, the role of FSTL1 in sepsis-induced acute kidney injury was disclosed for the first time within the present study, and TLR4/MyD88/NF- $\kappa \mathrm{B}$ signaling was proven to be involved in this process. Therefore, FSTL1 could be promising candidate for sepsis-induced acute kidney injury via inhibition of excessive inflammation and down-regulation of the TLR4/MyD88/NF- $\kappa$ B signaling pathway.

\section{AUTHOR CONTRIBUTIONS}

CS and AA designed the study, supervised the data collection. AK analyzed the data, interpreted the data. AbuA prepare the manuscript for publication and reviewed the draft of the manuscript. All authors have read and approved the manuscript.

\section{ETHICS APPROVAL AND CONSENT TO PARTICIPATE}

Ethical approval was obtained from the Ethics Committee of the First Affiliated Hospital of Xinjiang Medical University (Approval No. 20200301-09).

\section{ACKNOWLEDGMENT}

Thanks to all the peer reviewers for their opinions and suggestions.

\section{FUNDING}

This research received no external funding.

\section{CONFLICT OF INTEREST}

The authors declare no conflict of interest.

\section{AVAILABILITY OF DATA AND MATERIALS}

All data generated or analyzed during this study are included in this published article.

\section{REFERENCES}

[1] Rello J, Valenzuela-Sánchez F, Ruiz-Rodriguez M, Moyano S. Sepsis: a review of advances in management. Advances in Therapy. 2017; 34: 2393-2411.

[2] Dugar S, Choudhary C, Duggal A. Sepsis and septic shock: Guidelinebased management. Cleveland Clinic Journal of Medicine. 2020; 87: 5364.

[3] Gómez H, Kellum JA. Sepsis-induced acute kidney injury. Current Opinion in Critical Care. 2016; 22: 546-553.

[4] Mogensen TH. Pathogen recognition and inflammatory signaling in innate immune defenses. Clinical Microbiology Reviews. 2009; 22: 240273.

[5] Liu G, Zhao Y. Toll-like receptors and immune regulation: their direct and indirect modulation on regulatory CD4+ CD25+ T cells. Immunology. 2007; 122: 149-156.

[6] Vaure C, Liu Y. A comparative review of toll-like receptor 4 expression and functionality in different animal species. Frontiers in Immunology. 2014; 5: 316.

[7] Diomede F, Zingariello M, Cavalcanti MFXB, Merciaro I, Pizzicannella J, De Isla N, et al. MyD88/ERK/NFkB pathways and pro-inflammatory cytokines release in periodontal ligament stem cells stimulated by Porphyromonas gingivalis. European Journal of Histochemistry. 2017; 61: 2791 .

[8] Castoldi A, Braga TT, Correa-Costa M, Aguiar CF, Bassi ÊJ, Correa-Silva $\mathrm{R}$, et al. TLR2, TLR4 and the MyD88 signaling pathway are crucial for neutrophil migration in acute kidney injury induced by sepsis. PLoS ONE. 2012; 7: e37584.

[9] Mattiotti A, Prakash S, Barnett P, van den Hoff MJB. Follistatin-like 1 in development and human diseases. Cellular and Molecular Life Sciences. 2018; 75: 2339-2354.

[10] Chaly Y, Hostager B, Smith S, Hirsch R. Follistatin-like protein 1 and its role in inflammation and inflammatory diseases. Immunologic Research. 2014; 59: 266-272.

[11] Chaly Y, Marinov AD, Oxburgh L, Bushnell DS, Hirsch R. FSTL1 promotes arthritis in mice by enhancing inflammatory cytokine/chemokine expression. Arthritis and Rheumatism. 2012; 64: 1082-1088.

[12] Guo J, Liang W, Li J, Long J. Knockdown of FSTL1 inhibits oxLDLinduced inflammation responses through the TLR4/MyD88/NF- $\mathrm{BB}$ and MAPK pathway. Biochemical and Biophysical Research Communications. 2016; 478: 1528-1533.

[13] National Research Council Committee for the Update of the Guide for the $\mathrm{C}$, Use of Laboratory A. The national academies collection: reports funded by national institutes of health. Guide for the care and use of laboratory animals. Washington (DC): National Academies Press. 2011.

[14] Li Y, Zhang J, Su L, Kellum JA, Peng Z. Downregulation of TIMP2 attenuates sepsis-induced AKI through the NF- $\kappa b$ pathway. Biochimica et Biophysica Acta: Molecular Basis of Disease. 2019; 1865: 558-569.

[15] Luo C, Luo F, Zhang L, Xu Y, Cai G, Fu B, et al. Knockout of interleukin$17 \mathrm{~A}$ protects against sepsis-associated acute kidney injury. Annals of Intensive Care. 2016; 6: 56.

[16] Aziz M, Jacob A, Yang W, Matsuda A, Wang P. Current trends in inflammatory and immunomodulatory mediators in sepsis. Journal of Leukocyte Biology. 2013; 93: 329-342.

[17] Peters MMC, Meijs TA, Gathier W, Doevendans PAM, Sluijter JPG, Chamuleau SAJ, et al. Follistatin-like 1 in Cardiovascular Disease and Inflammation. Mini Reviews in Medicinal Chemistry. 2019; 19: 13791389.

[18] Zhou X, Xiao X, Huang T, Du C, Wang S, Mo Y, et al. Epigenetic 
inactivation of follistatin-like 1 mediates tumor immune evasion in nasopharyngeal carcinoma. Oncotarget. 2016; 7: 16433-16444.

[19] Fang Y, Zhang S, Li X, Jiang F, Ye Q, Ning W. Follistatin like-1 aggravates silica-induced mouse lung injury. Scientific Reports. 2017; 7 : 399.

[20] Su S, Zhang P, Zhang Q, Yin Z. GSK-3 $\beta$ inhibitor induces expression of the TLR4/MyD88/NF- $\kappa$ B signaling pathway to protect against renal ischemia-reperfusion injury during rat kidney transplantation. Inflammation. 2019; 42: 2105-2118.

[21] Yao H, Chi X, Jin Y, Wang Y, Huang P, Wu S, et al. Dexmedetomidine inhibits TLR4/NF- $\kappa$ B activation and reduces acute kidney injury after orthotopic autologous liver transplantation in rats. Scientific Reports. 2015; 5: 16849

[22] Qi M, Zheng L, Qi Y, Han X, Xu Y, Xu L, et al. Dioscin attenuates renal ischemia/reperfusion injury by inhibiting the TLR4/MyD88 signaling pathway via up-regulation of HSP70. Pharmacological Research. 2015; 100: 341-352.

[23] Chen F, Zhu X, Sun Z, Ma Y. Astilbin inhibits high glucose-induced inflammation and extracellular matrix accumulation by suppressing the TLR4/MyD88/NF- $\kappa$ B pathway in rat glomerular mesangial cells. Frontiers in Pharmacology. 2018; 9: 1187.

[24] $\mathrm{Hu} \mathrm{Y,} \mathrm{Pai} \mathrm{M,} \mathrm{Yeh} \mathrm{C,} \mathrm{Hou} \mathrm{Y,} \mathrm{Yeh} \mathrm{S.} \mathrm{Glutamine} \mathrm{administration}$ ameliorates sepsis-induced kidney injury by downregulating the highmobility group box protein-1-mediated pathway in mice. American Journal of Physiology Renal Physiology. 2012; 302: F150-F158.

[25] Zhong Y, Wu S, Yang Y, Li GQ, Meng L, Zheng QY, et al. LIGHT aggravates sepsis-associated acute kidney injury via TLR4-MyD88-NF$\kappa \mathrm{B}$ pathway. Journal of Cellular and Molecular Medicine. 2020; 24: 11936-11948.

How to cite this article: Chuanlong Song, Ayinuergul Adili, Adilijiang Kari, Abulaiti Abuduhaer. FSTL1 aggravates sepsis-induced acute kidney injury through regulating TLR4/MyD88/NF- $\mathrm{B}$ pathway in newborn rats. Signa Vitae. 2021;17(3):167-173. doi:10.22514/sv.2021.071. 